MATEC Web of Conferences 8, 04007 (2013)

DOI: $10.1051 /$ matecconf $/ 20130804007$

(c) Owned by the authors, published by EDP Sciences, 2013

\title{
Raman and fluorescence correlative microscopy in polarized light to probe local femtosecond laser-induced amorphization of the doped monoclinic crystal LYB:Eu
}

\author{
Nicolas Marquestaut, ${ }^{1}$ Marc Dussauze, ${ }^{2}$ Yannick Petit ${ }^{1,3, *}$ Arnaud Royon, ${ }^{1}$ Philippe Veber ${ }^{3}$, Véronique \\ Jubera, ${ }^{3}$ Michel Couzi, ${ }^{2}$ Vincent Rodriguez, ${ }^{2}$ Thierry Cardinal, ${ }^{3}$ and Lionel Canioni ${ }^{1}$ \\ ${ }^{1}$ Univ. Bordeaux, LOMA, UMR 5798, F-33400 Talence, France. \\ CNRS, LOMA, UMR 5798, F-33400 Talence, France. \\ ${ }^{2}$ Univ. Bordeaux, Institut des Sciences Moléculaires, CNRS UMR 5255, F-33400 Talence, France. \\ ${ }^{3}$ CNRS, ICMCB, UPR 9048, F-33608 Pessac, France. \\ Univ. Bordeaux, ICMCB, UPR 9048, F-33400 Pessac, France. \\ yannick.petit@u-bordeaux1.fr
}

An endless increasing number of laser/material interaction studies above laser-induced modification thresholds have been carried out over the past decade, either for undesirable laser-induced damages, for example in high power laser systems in relation with material ageing and material optical quality, or for profitable laser-induced alteration from direct laser writing (DLW) techniques. DLW is at the root of many applications such as the fabrication of micro-capillaries for micro-fluidics issues, waveguide writing, optical memories, etc... New functionalities based on enhanced optical property modifications can further be developed by tailoring the material chemical composition with photosensitive agents, in order to improve its interaction with the writing laser [1]. Thus, for the laserinduced linear properties, DLW can lead to plasmonic enhancement from nanoparticles [2], fluorescence from metallic clusters [3] or oxidation degree modifications of rare earth ions [4]. For the nonlinear properties, second-harmonic generation (either from locally buried electric field [5] or from locally laser-induced micro-crystallization [6]) or third-harmonic generation [7] can be obtained.

Most of the studies are related to isotropic material modifications (density change, oxidation degree changes, local crystallization, etc...) [1] while much less refer to laser-induced modifications inside crystals, being isotropic [8] or not [9]. Still, laser-induced modifications inside a crystal matrix are promising research topics for perennial optical devices and applications, thanks to the intrinsic stability of the crystalline matrix arrangement, but also for waveguide applications for efficient laser and Raman amplification as well as for frequency conversion. Generally, laser-induced modifications lead to local index change, local melt, local amorphization or even local recrystallization, giving rise to differential optical properties with respect to the non-irradiated bulk matrix.

In this work, we have studied the local modification induced by a femtosecond (fs) near infrared NIR laser on a monoclinic crystal matrix LYB, highly doped with $\mathrm{Eu}^{3+}$ rare earth ions. Thanks to point-bypoint optical mapping in both Raman and fluorescence micro-spectroscopy in polarized light, we report on the local changes corresponding to partial amorphization of the LYB:Eu crystal, resulting to significant reshaping of the spectral properties in polarized light. The optical specificities of lowsymmetry crystal optics were properly taken into account, to provide meaningful interpretation of the modified Raman and fluorescence spectra. Strong laser-induced amorphization of the crystal matrix have been evidenced. An accurate characterization of the spectral variations of spatial distribution have shown (i) an homogeneous degree of amorphization within a volume at least two times larger than the laser-structuring voxel, and (ii) a variation of density within the amorphous region.

Such study shows that both Raman and fluorescence spectroscopies are relevant tools to probe soft laser-induced modifications and related localized phase transitions, which opens interesting potentiality to further identify the fundamental modifications of the LYB:Eu crystal that could behave

This is an Open Access article distributed under the terms of the Creative Commons Attribution License 2.0, which permits unrestricted use, distribution, and reproduction in any medium, provided the original work is properly cited. 
as the elementary brick of photonics applications. Since the LYB:Eu is a potential laser crystal [10], these observations are of strong interest when considering possibilities to tune both structural and optical properties of this material by direct laser writing, especially for weaker crystal modifications related to waveguide writing for laser amplification applications [11-15]. Moreover, since such crystal is a low symmetry crystal, intensity thresholds for laser-induced modifications should significantly depend on the considered crystal orientation [16]. Finally, such correlative approach provides a versatile tool for micro-spectroscopy that can also apply for advanced imaging microscopy to probe wanted DLW but also undesired laser-induced damages, in the case of laser material ageing.

\section{$\underline{\text { References and links }}$}

1. A. Royon, Y. Petit, G. Papon, M. Richardson, and L. Canioni, "Femtosecond laser induced photochemistry in materials tailored with photosensitive agents [Invited]," Optical Materials Express 1, 866-882 (2011).

2. A. Podlipensky, A. Abdolvand, G. Seifert, and H. Graener, "Femtosecond laser assisted production of dichroitic 3D structures in composite glass containing Ag nanoparticles,” Appl. Phys., A Mater. Sci. Process. 80(8), 1647-1652 (2005).

3. M. Bellec, A. Royon, B. Bousquet, K. Bourhis, M. Treguer, T. Cardinal, M. Richardson, and L. Canioni, "Beat the diffraction limit in 3D direct laser writing in photosensitive glass," Optics Express 17, 10304-10318 (2009).

4. J. Qiu, K. Miura, T. Suzuki, T. Mitsuyu, and K. Hirao, "Permanent photoreduction of $\mathrm{Sm}^{3+}$ to $\mathrm{Sm}^{2+}$ inside a sodium aluminoborate glass by an infrared femtosecond pulsed laser," Appl. Phys. Lett. 74(1), 10-12 (1999).

5. J. Choi, M. Bellec, A. Royon, K. Bourhis, G. Papon, T. Cardinal, L. Canioni, and M. Richardson, "Three-dimensional direct femtosecond laser writing of second-order nonlinearities in glass," Optics Letters 37, 1029-1031 (2012).

6. K. Miura, J. Qiu, T. Mitsuyu, and K. Hirao, "Space-selective growth of frequency-conversion crystals in glasses with ultrashort infrared laser pulses,” Opt. Lett. 25, 408 (2000).

7. L. Canioni, M. Bellec, A. Royon, B. Bousquet, and T. Cardinal, "Three dimensional optical data storage using third-harmonic generation in silver zinc phosphate glass," Optics Letters 33, 360-362 (2008).

8. K. Kawamura, M. Hirano, T. Kurobori, D. Takamizu, T. Kamiya, and H. Hosono, "Femtosecond-laserencoded distributed-feedback color center laser in lithium fluoride single crystals,” Appl. Phys. Lett. 84, 311 (2004)

9. V. Apostolopoulos, L. Laversenne, T. Colomb, C. Depeursinge, R. P. Salathé, M. Pollnau, R. Osellame, G. Cerullo, and P. Laporta, "Femtosecond-irradiation-induced refractive-index changes and channel waveguiding in bulk $\mathrm{Ti}^{3+}$ :Sapphire,” Appl. Phys. Lett. 85, 1122 (2004).

10. R. Reisfeld, R. A. Velapoldi, L. Boehm, and M. Ish=Shalom, Transition Probabilities of Europium in Phosphate Glasses," J. of Physical Chemistry, 75 (1971) 3980-3983.

11. R. Cattoor, I. Manek-Hönninger, J.-Ch. Delagnes, Y. Petit, B. Bousquet, V. Jubera, A. Fargues, Ph. Veber, M. Velazquez, A. Garcia, and L. Canioni, "Potential of the Eu:LYB crystal as a laser material for DPSS lasers emitting at $613 \mathrm{~nm}$,” Proc. SPIE 8235, 82351A (2012).

12. F. Bain, A. Lagatsky, R. Thomson, N. Psaila, N. Kuleshov, K. Kar, W. Sibbett and C. Brown, "Ultrafast laser inscribed $\mathrm{Yb}: \mathrm{KGd}\left(\mathrm{WO}_{4}\right)_{2}$ and $\mathrm{Yb}: \mathrm{KY}\left(\mathrm{WO}_{4}\right)_{2}$ channel waveguide lasers". Optics express, 17, 22417-22422 (2009).

13. J. Siebenmorgen, K. Petermann, G. Huber, K. Rademaker, S. Nolte and A. Tünnermann, "Femtosecond laser written stress-induced $\mathrm{Nd}: \mathrm{Y}_{3} \mathrm{Al}_{5} \mathrm{O}_{12}$ (Nd:YAG) channel waveguide laser" Applied Physics B, 97 251-255 (2009).

14. S. Beecher, R. Thomson, D. Reid, N. Psaila, M. Ebrahim-Zadeh and A. Kar, "Strain field manipulation in ultrafast laser inscribed $\mathrm{BiB}_{3} \mathrm{O}_{6}$ optical waveguides for nonlinear applications" Optics letters, 36 $4548-4550$ (2011).

15. A. Ródenas, A. Nejadmalayeri, D. Jaque and P. Herman, "Confocal Raman imaging of optical waveguides in $\mathrm{LiNbO}_{3}$ fabricated by ultrafast high-repetition rate laser-writing". Optics express, 16 13979-13989 (2008).

16. Y. Petit, S. Joly, P. Segonds, and B. Boulanger, "Recent advances in monoclinic crystal optics," Laser \& Photonics Reviews, (2013) to appear in press. 